\title{
Rosmarinus Officinalis $L$. para o tratamento da ansiedade: uma revisão de literatura
}

\author{
Rosmarinus Officinalis $L$. for the treatment of anxiety: a literature review \\ Rosmarinus Officinalis $L$. para el tratamiento de la ansiedad: revisión de la literatura
}

Recebido: 20/08/2021 | Revisado: 27/08/2021 | Aceito: 30/08/2021 | Publicado: 01/09/2021

\author{
Adelma do Socorro Gonçalves Pimentel \\ ORCID: https://orcid.org/0000-0003-0048-4976 \\ Universidade Federal do Pará, Brasil \\ E-mail: adelmapi@ufpa.br
}

\begin{abstract}
Resumo
A ansiedade comum e as formas psicopatológicas são tratadas incluindo remédios alopáticos, o que pode ocasionar dependência do uso de medicamentos. Assim, neste texto apresentamos o enfoque fenomenológico em psicopatologia e a contribuição do Rosmarinus Officinalis no tratamento integral da ansiedade, por meio de uma revisão sistemática de literatura descrevendo pesquisas que incluem o Rosmarinus Officinalis no tratamento da ansiedade nas formas de tintura, chá, e óleo essencial como moderador conjunto a redução do cansaço físico e mental. Os procedimentos envolvem coleta nas bases de dados nacionais, período de 2010 a 2020. Destaca-se entre os resultados que os estudos clínicos e observacionais do Rosmarinus Officinalis são mais realizados com modelos animais; contudo demonstram potencial terapêutico no tratamento da ansiedade. Considera-se que agregar o mesmo em projetos psicoterapêuticos sistemáticos de base fenomenológica existencial favorece a melhora da concentração pessoal, a respiração e a delimitação do sentido que a ansiedade tem na vida emocional e social das pessoas.
\end{abstract}

Palavras-chave: Ansiedade; Psicopatologia fenomenológica; Rosmarinus Officinalis.

\begin{abstract}
Common anxiety and psychopathological forms are treated including allopathic remedies, which can lead to drug dependence. Thus, in this text we present the phenomenological focus on psychopathology and the contribution of Rosmarinus Officinalis in the integral treatment of anxiety, with narrative review literature describing research that includes Rosmarinus Officinalis in the treatment of anxiety in the forms of tincture, tea, and essential oil as a joint moderator to reduce physical and mental fatigue. The procedures involve collection in national databases, from 2010 to 2020. Among the results, it is noteworthy that clinical and observational studies of Rosmarinus Officinalis are more carried out with animal models; however, they demonstrate therapeutic potential in the treatment of anxiety. It is considered that adding the same, in systematic psychotherapeutic projects with an existential phenomenological basis, favors the improvement of personal concentration, breathing and the delimitation of the meaning that anxiety has in people's emotional and social lives.
\end{abstract}

Keywords: Anxiety; Phenomenological psychopathology; Rosmarinus Officinalis.

\section{Resumen}

Se tratan las formas comunes de ansiedad y psicopatológicas, incluidos los remedios alopáticos, que pueden conducir a la drogodependencia. Así, en este texto presentamos el enfoque fenomenológico de la psicopatología y la contribución de Rosmarinus Officinalis en el tratamiento integral de la ansiedade, por medio de revisión narrativa de la literatura que describe investigaciones que incluyen Rosmarinus Officinalis en el tratamiento de la ansiedad en forma de tintura, té y aceite esencial como moderador conjunto para reducir la fatiga física y mental. Los procedimientos involucran recolección en bases de datos nacionales, de 2010 a 2020. Entre los resultados, se destaca que los estudios clínicos y observacionales de Rosmarinus Officinalis se realizan más con modelos animales; sin embargo, demuestran potencial terapéutico en el tratamiento de la ansiedad. Se considera que sumar lo mismo, en proyectos psicoterapéuticos sistemáticos con base fenomenológica existencial, favorece la mejora de la concentración personal, la respiración y la delimitación del significado que tiene la ansiedad en la vida emocional y social de las personas.

Palabras clave: Ansiedad; Psicopatología fenomenológica; Rosmarinus officinalis.

\section{Introdução}

Nesta escritura apresentamos uma revisão de literatura sobre o potencial do Rosmarinus Officinalis (RO) aplicado ao tratamento da ansiedade. Abre-se a reflexão apresentando excerto de dois casos clínicos no contexto da ansiedade. No ano de 2014 Marina, durante uma reunião com o Diretor de Recursos Humanos da empresa em que ela trabalhava lhe disse: Todos 
sabem que você é ansiosa. Em 2018, durante uma sessão psicoterápica virtual breve comigo, Marina me contou que percebeu que a frase fora proferida pelo Chefe como uma ofensa, ante a pressa por ela demonstrada na reunião de planejamento de implantação de um serviço. Marina se considerava proativa, não se sentia ansiosa. Ela me comunicou que pensou, após escutar o hipotético insulto: para que ele quer me ofender? Talvez para esconder o que os outros pensam dele. Desde quando buscar soluções as tarefas da empresa têm a ver com a ansiedade. Até aquele momento, Marina e o Diretor riam juntos dos outros funcionários; contudo, daquele dia em diante a conversação hílare entre os dois findou.

Em março de 2020 quando foi decretada no Brasil a pandemia da COVID-19, Lauro estava com seu pai internado em hospital particular por câncer de próstata. Ele passou a desconfiar da possibilidade de que, todos a sua volta pudessem transmitir-lhe o Corona vírus. Lauro sentia-se muito ansioso, em pânico, com pavor de ser acometido pela COVID-19 e morrer antes de seu pai. Durante o dia permanecia no hospital até ser substituído pelo irmão; rapidamente voltava a proteção de sua casa, na qual habitava sozinho, tomava um banho, descartava as roupas sujas e ia dormir. Como tinha que cuidar do pai, Lauro fazia um esforço enorme para reduzir seu sofrimento psíquico.

Marina e Lauro em 2020 iniciaram a psicoterapia breve gestáltica comigo. Ela fora informalmente "nomeada" de ansiosa por alguém, não inserido na área da saúde mental; enquanto Lauro que vivenciava a ansiedade obteve Diagnóstico de um neurologista, dando início a um tratamento por trintas dias com Venlaxin, (37,5mg em uma dose diária). Ambos transitavam em um contexto psíquico existencial, em que a ansiedade era uma forma de manifestar o sofrimento vivido pautado nas dificuldades ontológicas na vivencia de cuidar e não estar sendo cuidado por alguém.

Em nosso trabalho sobre a ansiedade adotamos uma abordagem fenomenológica do cuidado em saúde, configurada ontologicamente pela elaboração de Heidegger (2013). Para o filósofo, o cuidado (em alemão Sorge) que contribui para a ampliação da consciência intencional é o expresso na forma de solicitude e preocupação com o outro: "Cuidar é ser-no-mundo e ser-com-o-outro". Aplicando a premissa ao atendimento de Laura e Marina, em uma dimensão teórica ideal, a caminhada de ambos permeada pela a dor e o sofrimento, tem possibilidade de seguir adiante, ou seja, transcender a facticidade que transforma nossas vidas. (Pimentel, Castro \& Miranda, 2019).

Em nossa apreensão fenomenológica existencial da ansiedade agregamos uma crítica aos enquadres diagnósticos que oferecem conforto aos psiquiatras e psicólogos durante a avaliação clpinica baseada no isolamento de categorias de doenças. (Andrade, et al, 2019). Compreendemos ainda, a gênese da ansiedade como decorrência da cultura, ou seja, da presença do descuido e do cansaço em cuidar que algumas pessoas vivenciam, devido a afetação do sentido da existencial, ou exaustão emocional. Ressaltamos que, estudar a ansiedade nesta dimensão, não alude em "negacionismo" das implicações biológicas e genéticas nas formas patológicas.

Alain Ehrenberg produziu uma trilogia que analisa processos de sociabilidade e subjetivações do humano nos cenários de governo público e privado: Le Culte de la Performance, (1991); O indivíduo incerto (1995); e o Cansaço de ser você mesmo (1998) As reflexões denotam as exigências que as pessoas estão submetidas para realizarem ajustamentos normatizados pela economia, beleza, e pela expressividade digital. Neste bojo, resulta desta cultura uma imensa abertura para a vivencia de estados de ansiedade.

Para Kátia Barbosa Macedo (2011) há um imenso sentimento de desamparo das pessoas na modernidade, marcado por desenraizamento dos vínculos, flutuação da identidade, conforme a moda situacional, tedio, fragilidade emocional,

As psicopatologias emergem quando faltam ao sujeito os meios habituais, ou seja, culturalmente codificados e legitimados para lidar com os conflitos derivados das imposições do tipo psicológico ideal. A psicopatologia é produto de um entrave no processo de socialização, a cultura pode ser um fator patogênico, não porque produz um tipo particular de identidade étnica, mas porque é um elemento causal na cadeia patogênica, quando produz uma dissimetria entre as exigências sociais e os meios adequados para cumpri-las. (p 99-100) 
Sobre a incidência da ansiedade no Brasil em pesquisa virtual sobre saúde mental, o Ministério da saúde em 2020, com uma amostra de 17.491 indivíduos, idade média de 38,3 anos, variando entre 18 e 92 anos, maioria do sexo feminino (71,9\%), de raça/cor autorreferida branca $(61,3 \%)$, casados $(44,3 \%)$, residentes em bairros populares $(46,8 \%)$; renda mensal variando entre $\mathrm{R} \$ 1.049,00$ e $\mathrm{R} \$ 2.096,00$ (24,3\%); identificou que a ansiedade teve proporção de 86,5\%; transtorno de estresse pós-traumático (45,5\%); e uma baixa proporção de depressão (16\%) em sua forma mais grave. (Pesquisa MS, 2020)

De acordo com Melo, Nogueira, Macedo \& Macedo (2020, p 5302) "No Brasil, a prevalência dos transtornos de ansiedade constitui-se em mais de $9 \%$ da população, totalizando mais de 18 milhões de pessoas". Os autores pesquisaram em prontuários ativos e não ativos de pacientes a prevalência de transtorno de ansiedade no município paraense de Santarém, região Norte Amazonia, e identificaram que "As variáveis predominantes: são as mulheres, com iguais resultados para o estado civil de casados (as) e solteiros (as). A idade adulta obteve maior frequência, sendo os jovens adultos os mais acometidos". (p.5309).

Quanto aos medicamentos mais usados para o tratamento da ansiedade, nos manuais de psiquiatria encontram-se descritos os Benzodiazepínicos: Alprazolam, Clordrazepoxida, Clonazepam, Diazepam, lorazepam, Oxazepam, amplamente prescritos ao Transtorno de Ansiedade Generalizada, Pânico e Fobias. Por sua vez, os grandes laboratórios farmacêuticos pesquisam medicamentos "eficazes" no controle dos sintomas. Para o tratamento do Transtorno da Ansiedade Generalizada (TAG) tem-se indicação do Ansitec/Cloridrato de Buspirona, com dosagem inicial de $15 \mathrm{mg}$ diários por seis meses (5mg, 3 vezes dia, de preferência nos intervalos das refeições" (www.libbs.com.br ).

Andreatini, Boergen-Lacerda e Filho (2001) mencionam que a produção da Buspirona "revolucionou" o tratamento das TAG. Porém, considero que não se constitui uma "revolução" do tratamento a exclusão dos aspectos subjetivos da vivencia da ansiedade, em que as pessoas configuram sínteses originais das suas vivencias dos sintomas nas "doenças" mencionadas. Destarte, a ansiedade não pode ser reduzida a tratamentos que medem e/ou validam, apenas a eficácia dos medicamentos.

A relevância social e cientifica deste trabalho ancora-se em diversos fundamentos: a pesquisa, em pleno curso denominada, Clínica interdisciplinar da ansiedade em adultos pela psicoterapia breve gestáltica e uso do Rosmarinus Officinalis - em capsulas e gotas, aprovada pelo Comitê de ética do Hospital das Clínicas do Pará, sob número do Parecer: 4.901.293; a articulação entre diálogos interdisciplinares dos saberes da Psicologia, Psiquiatria, Farmácia, Nutrição e Fitoterapia, com os saberes tradicionais das comunidades acerca das contribuições das plantas medicinais a ansiedade. Bem como, ensejamos contribuir para que fitoterápicos deixem de ser considerados pela ciência psicológica e psiquiátrica como "opções na área de bem-estar"; "complementares". Três importantes motivos para a prioridade: a) o limite da eficácia do tempo dos remédios alopáticos; b) considerar a vivencia e a subjetividade; c) os efeitos colaterais dos alopáticos, pendencias permanentes no tratamento da ansiedade, em face a provocarem sonolência, alteração motora, interações medicamentosas e dependência física e psicológica. (Andreatini, Boergen-Lacerda \& Filho, 2001).

\section{Metodologia}

Revisão narrativa de literatura produzida no período de 2001 a 2021 coletada nas bases de dados do Google Acadêmico, Repositórios Institucionais, Scielo e Bireme. Buscou-se identificar as contribuições do Rosmarinus Officinalis ao tratamento das formas de ansiedade pela inclusão de trabalhos empíricos e de revisão nas formas de conclusão de curso, dissertações de mestrado e doutorado nas áreas de saúde; campo em que, usualmente as áreas de produção de pesquisas são a farmácia, a odontologia, a psicologia. Também incluímos materiais sobre projetos de farmácias vivas que cultivam plantas medicinais, desenvolvidos por instituições estaduais brasileiras. Os descritores em Ciências da Saúde (DECS/MESH) foram: 
ansiedade and. sofrimento psíquico; ansiedade and fenomenologia. Encontramos 17 materiais dispostos nos anos de 2001 a 2021.

Figura 1. Distribuição de artigos por ano.

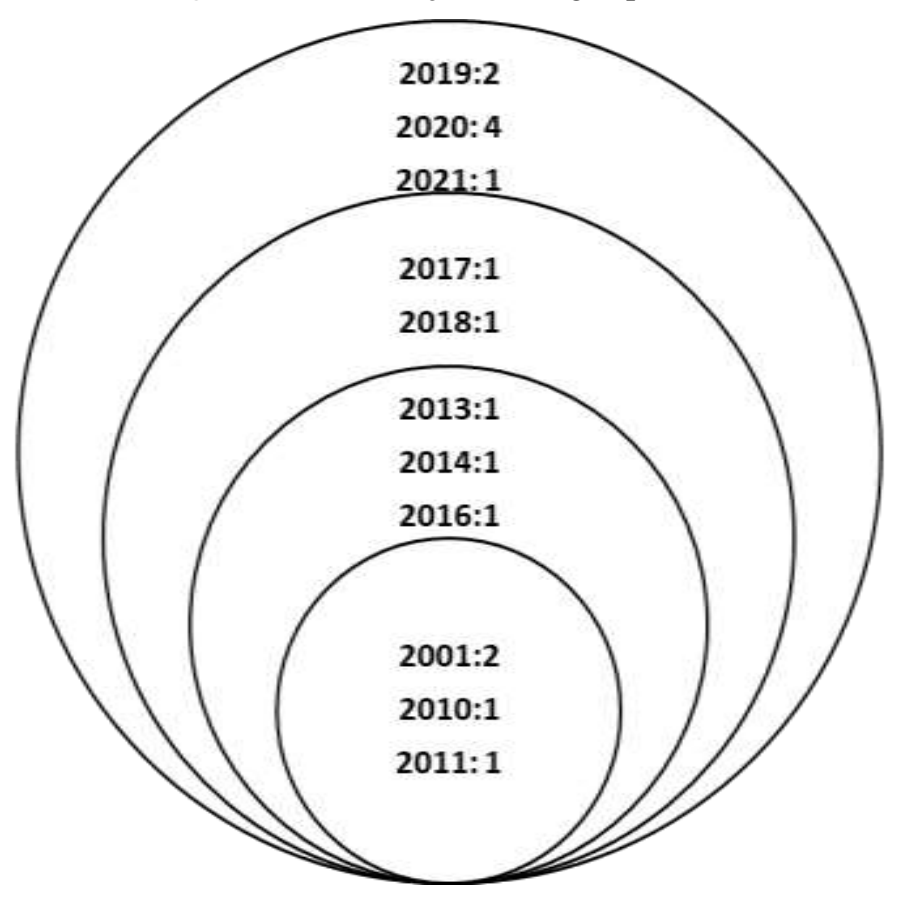

Fonte: Autora.

\section{Resultados e Discussão}

Os usos, aplicações e o contexto do RO no tratamento da ansiedade em Saúde Mental foram estudados por Evangelista, uma farmacêutica que, em 2019 escreveu sua monografia de graduação compilando publicações no período de 2009 a 2019. Além disso, a autora criticou tratamentos unilaterais, baseados na prescrição de medicamentos que alteram a bioquímica do organismo. Ela avaliou que se deve tratar a ansiedade multidisciplinarmente para obter mais eficácia nos “Tratamentos ansiolíticos e antidepressivos. Considerar imprescindivelmente os aspectos psicológicos, biológicos e sociais do paciente, como um todo. O tratamento poderá utilizar a psicoterapia, mudanças no estilo de vida e a terapia farmacológica, quando necessária.” (Evangelista, 2019, p 18)

A crítica se alinha a nossa concepção de tratamentos da ansiedade, em que analisamos relevante buscar terapêuticas fenomenológicas existenciais que abordem a vivencia da ansiedade e a singularidade de sua manifestação na existência dos clientes, e não apenas aplicar medicamentos das,

Classes de fármacos como os benzodiazepínicos, antagonistas betas adrenérgicos, inibidores seletivos da recaptação de serotonina e inibidores da recaptação da serotonina-norepinefrina, os principais tratamentos farmacológicos utilizados atualmente no tratamento da ansiedade. Entretanto, devido aos diversos efeitos indesejáveis podem ser causados aos pacientes, incluindo quadros de tolerância e dependência medicamentosa. (Evangelista, 2019, p 18).

Conforme a farmacêutica Evangelista, o uso mais conhecido e habitual do RO se dá pela inalação; entretanto, abrange a aplicação na pele na forma de cremes, e a ingestão de cápsulas e rasuras. Fundamental nos modos de aplicação do RO por profissionais de saúde a ansiedade, com menção especial a Psicóloga clínica - em cuja graduação, frequentemente não há 
atividades curriculares que contenham estudos sobre a farmacocinética do R. officinalis - é necessário estudar e trabalhar interdisciplinarmente para conhecer, também, os possíveis efeitos tóxicos da planta, quando administrada por via oral, já que “As moléculas dos Óleos Essenciais são absorvidas no intestino e distribuídas para os tecidos. Alguns são tóxicos e outros possuem substâncias muito potentes em baixas dosagens, a indicação dessa via de administração deve ser feita com bastante cautela. (Evangelista, 2019, p. 32)

Embora o RO seja apresentado nas pesquisas como fitoterápico de ações "Farmacológicas, atribuídas pelo uso popular, como: hipoglicêmico, antiaterogênico, anti-hipertensivo, hipocolesterolêmico, antioxidante, anti-hipertensivo, antiinflamatório, hepatoprotetor, antidepressivo, antiproliferativo e antibacteriano" (Baiotto, Tremêa \&, Colet, 2020); é imprescindível realizar estudos sistemáticos e densos sobre a aplicação no tratamento da ansiedade,

No Brasil, a fitoterapia é pouco utilizada na atenção básica, possivelmente devido ao fato de fitoterápicos para TAG não serem disponibilizados pelo SUS, conforme a Relação Nacional de Medicamentos Essenciais (RENAME). As principais plantas medicinais, com efeitos ansiolíticos, indicadas pelo Ministério da Saúde em 2016 são, Passiflora incarnata L., Valeriana officinalis L., Matricaria chamomilla L., Hypericum perforatum L., Melissa officinalis L. e Piper methysticum G. Forst.” (Zeni; Liz; Duarte; Zeni, 2021).

De acordo com Souza e Silva (2018), os fitoterápicos associados ao tratamento dos distúrbios do sono, ansiedade e depressão leve presentes na Lista de Fitoterápicos de Registro Simplificado são: "Hypericum perforatum, indicado como antidepressivo leve; Kava-kava (Piper methysticum) para ansiedade e insônia, sendo o seu uso máximo por até dois meses; Valeriana officinalis como sedativo moderado, hipnótico e no tratamento de distúrbios do sono associados à ansiedade; ErvaCidreira (Melissa officinalis) como carminativo, antiespasmódico e ansiolítico leve e Maracujá (Passiflora edulis) como ansiolítico leve. (http://conic-semesp.org.br/anais/files/2018/trabalho-1000000560.pdf)

Em Baiotto, Tremêa, e Colet (2020, p3) são descritos os componentes ativos do RO solúveis em ágia: “Ácidos hidroxicinamicos e flavonoides glicosilados, enquanto os diterpenos de abietano, como ácido carnosico, carnosol ou rosmadial e outros compostos hidrofobicos, como flavonas metiladas genkwanin e cirsimaritina, e a flavona hesperetina são abundantes no extrato não solúvel em água". Quanto a dosagem, os autores mencionam na sua pesquisa um outro estudo apresentando, "Que não foram encontradas diferenças entre as doses terapêuticas dos extratos de alecrim de 100 e $250 \mathrm{mg}$, indicando um efeito platô a partir de $100 \mathrm{mg}$, sendo que em duas semanas de uso, os efeitos positivos do consumo da planta foram notados" (Baiotto, Tremêa, \& Colet, 2020, p.4).

Lima;Heck; Barbieri; Kaster e Lopes (2016) analisaram os produtos do "Grupo de agricultoras Esperança: Saúde Alternativa: oito elixires, dois xaropes e seis pomadas, a partir de 45 plantas medicinais, mel, própolis, cera de abelha, breu e vaselina" (p 3322). Na atividade das agricultoras o uso do Rosmarinus Officinalis foi associado ao tratamento de "problemas estomacais, em dosagem de 20 gotas, 3 vezes ao dia, antes das refeições" (p.3323). Em referência a demandas clínicas em saúde mental, as autoras apontam outro produto: o Elixir dos Nervos, "Composto pelas Tinturas de cassaú (s.i); jurubeba (Solanun sp.), maracujá (Passiflora sp.) e mulungu (Erythrina speciosa) para o nervosismo, angústia e insônia, ministrado em dispensação de "20 gotas, 3 vezes ao dia, após as refeições" (P 3323).

Arruda, (2014), em pesquisa para o Mestrado em Psicologia da Saúde da Faculdade da Saúde, da Universidade Metodista de São Paulo, descreveu as formas tradicionais de utilização dos óleos essenciais: por aromatização ambiental nas habitações e consultórios clínicos; e na pessoa por inalação, massagens, banhos aromáticos.

Quanto ao potencial terapêutico na esfera do Sistema Nervoso, informou que "Os óleos essenciais podem tanto estimular como deprimir a atividade neuronal. Os estimulantes têm potencial terapêutico para atuar em transtornos de depressão, na fadiga, em distúrbios de memória; os depressores da atividade cerebral podem auxiliar no combate à insônia e estados de ansiedade como nervosismo e histeria" (Arruda, 2014, p 6). 
Especificamente sobre o RO elencou pesquisas que indicam:

Efeito antimicrobiano (compostos $\alpha$-pineno e 1,8-cineol); ação anti-inflamatória e antinociceptiva periférica: administrado por via oral em várias doses $(250,500$ e $750 \mathrm{mg} / \mathrm{kg}$ ) ação na depressão, em camundongos mostrou que o extrato hidroalcóolico de RO (em doses variando de 1 a $300 \mathrm{mg} / \mathrm{kg}$ administrado por via oral, de forma aguda ou repetida) foi efetivo em dois modelos de depressão, o de natação forçada e o de suspensão pela cauda. Em humanos, o óleo essencial de RO promoveu mudanças assimétricas no eletroencefalograma de adultos e crianças, mudanças essas compatíveis com estados positivos de humor, sugerindo um potencial antidepressivo dessas substâncias" (Arruda, 2014, p.11).

Dalmarco (2012) em sua tese de doutorado em química pela Universidade Federal de Santa Catarina pesquisou as propriedades químicas e biológicas de RO $l$. em ratos; fundamentando sua justificativa de que,

São inúmeros os exemplos de medicamentos desenvolvidos direta ou indiretamente de fontes naturais, sobretudo das plantas. Destacam-se a morfina, a pilocarpina, os digitálicos, os curares, a quinina, a artemisinina, a atropina, a escopolamina, as estatinas, o cromolin, os fármacos usados no tratamento de câncer (vimblastina, vincristina, taxol, campotecinas), os imunossupressores, os antibióticos, os inibidores da enzima conversora de angiotensina, como o Captopril (p.22).

A pesquisa da autora, embora voltada para o tratamento da depressão e concretizada com ratos, aponta o potencial do RO; além de apresentar os componentes terapêuticos da planta: a cânfora, encontrada no RO espanhol; o cineol/eucaliptol nas plantas francesas, inglesas e indianas; e a cetona verbenona exclusivamente na francesa. Há uma riqueza de informações nos resultados do estudo, por exemplo, a validação farmacológica e terapêutica do RO nas formas de óleo essencial; e a "Evidência para o princípio ativo de alto teor de cineol que apresenta efeito anestésico, antisséptico, bactericida, expectorante, muito utilizado na indústria farmacêutica." (Dalmarco, 2012, p.58).

É significante referir que, a pesquisa descreve os efeitos antidepressivos do extrato bruto hidroalcóolico do RO, "No tratamento agudo dos camundongos com o extrato por via oral; reduziu significativamente o tempo de imobilidade no teste do nado forçado (na dose de $100 \mathrm{mg} / \mathrm{kg}$ ) e da suspensão em cauda (nas doses de 10 e $100 \mathrm{mg} / \mathrm{kg}$ ), quando comparado com o grupo controle sem alterações". (Dalmarco, 2012, p 107-108)

Outra importante conclusão que a autora elenca é sobre a duração do efeito do RO: "O estudo antidepressivo do extrato bruto, frações, compostos isolados e óleo essencial da Rosmarinus officinalis, demonstraram resultados comparados aos apresentados pela fluoxetina, com destaque para o extrato bruto, ácido ursólico e óleo essencial, o que sugere a possibilidade de utilização da planta no tratamento da depressão" (Dalmarco, 2012 p 112).

Por sua vez, Lima (2010, p 4) elaborou dissertação de mestrado em Farmacologia na UNESP com o objetivo de examinar a potencialidade do RO no tratamento da depressão e da ansiedade em modelos animais. Embora o fitoterápico seja uma possibilidade para intervenções clínicas, o trabalho contém uma base epistemológica clássica ancorada na explicação "De teorias psicológicas e biológicas desenvolvidas para se entender o substrato neuroquímico na etiologia da depressão. A hipótese que sugere que o déficit funcional de um ou mais sistemas de neurotransmissores (noradrenalina, dopamina e serotonina) em certas sinapses envolve o processo patológico da depressão tem sido de grande interesse e investigação" (p.2)

Do material produzido me atenho, unicamente, as referências sobre a ansiedade relacionadas ao RO. A articulista afirmou que "Dados etnofarmacológicos, indicam o uso de diferentes preparações de Rosmarinus officinalis L. por sua atividade sobre o Sistema Nervoso Central, que vão da sedação à estimulação.” Os resultados obtidos durante a avaliação da atividade ansiolítica "No Labirinto em Cruz Elevado associados à ansiedade são a frequências de entradas e o tempo de permanência nos braços abertos, considerados de modo relativo ao total de entradas e ao tempo total de permanência no Labirinto. Após o tratamento com OE não se observam diferenças significantes entre os grupos tratados com OE e o 
grupo Tween.” (Lima, 2010, p 27)

No estudo de Gouveia e Siminonato (2019) do Memento fitoterápico para prática clínica na Atenção Básica de Santa Catarina (2019), o RO é referenciado na categorização de: "Plantas para dor", sendo usadas as Folhas e flores frescas e secas". $\mathrm{E}$ as indicações de plantas para o transtorno de humor são: Hypericum perforatum L. (hiperico); Lippia alba (Mill.) (N.E. Br. ex Britton \& P. Wilson (salva), p.55; Melissa officinalis L. (melissa), (p. 57); Passiflora sp. (maracujá), e Valeriana officinalis L. (valeriana) (p.59)

Na revisão sistemática de literatura do RO, Baiotto: Tremêa \& Colet, (2020, p 3) apontam que, "O perfil polifenólico dessas plantas e caracterizado pela presença de ácido carnósico, carnosol, ácido rosmarínico e hesperidina, como componentes principais e responsáveis pelos principais efeitos farmacológicos que são ações anti-inflamatórias, antivirais e antibacterianas". Por sua vez, Penteado e Cecy descrevem os efeitos do RO, "Por suas virtudes tônicas e estimulantes, atua sobre o sistema nervoso (cansaço mental) e cansaço físico, antidepressivo natural, carminativo e vasodilatador." (http://www.unieuro.edu.br/sitenovo/revistas/downloads/farmacia/cenarium_02_02.pdf).

Finalizamos pontuando que Pagani e Silva (2017) realizaram estudo de plantas com propriedades calmantes cultivadas no horto da cidade de Urupema-SC abrangendo o Rosmarinus officinalis L.; Cymbopogon citratus; loysia triphylla; Hypericum perforatum L. e Melissa officinalis L. As autoras assinalam que o RO é estimulante, uma dentre suas propriedades associadas ao tratamento da ansiedade, atuando sobre o sistema nervoso no cansaço mental físico. Também criticam as diferenças na prescrição de alopáticos e de fitoterápicos no tratamento da ansiedade, muitas vezas reduzindo as plantas medicinais como um artifício "paliativo".

\section{Considerações Finais}

No escopo do texto abordamos o uso e/ou a aplicação do RO no tratamento da ansiedade comum e na forma psicopatológica. Visamos com a pesquisa contribuir para que equipes de saúde possam se valer, na atenção básica de estratégia psicoterapêutica, fenomenológica e fitoterápica. Considera-se que, nestas formas de adoecimento, os pensamentos ruminantes, a raiva acumulada e os deslocamentos da temporalidade presente para o futuro mantem a pessoa em um círculo vicioso de sofrimento psíquico. Em nosso trabalho clínico percebemos que a vivencia da ansiedade se organiza na polaridade controle/descontrole, em que os pensamentos irracionais adquirem a direção dos movimentos e das relações da pessoa, dada a impossibilidade percebida de saborear a vida pelo curso dos pensamentos únicos: "vou morrer". Com a instalação do descontrole das forças pessoais, do entorno familiar e no trabalho, abre-se a possibilidade da vivencia da ansiedade afetar a motivação, instalar o desanimo, aumentar o acumulo de situações desagradáveis no pensamento criando um emaranhado crônico de sensação de incapacidade e limitação ao agir.

Considerando que o uso do Rosmarinus Officinalis é socialmente valioso pela aplicação nas áreas psicológicas, psiquiátrica e nutricional nosso programa de pesquisa intervenção em psicoterapia é promissor. De modo amplo, enfocamos no tratamento das formas de ansiedade, as intenções, os sentimentos e as reações pessoais derivadas das regulações da vida social que operam para controlar a autonomia e a crítica as fontes causadoras da opressão social. Os suportes pessoais ampliados favorecem a pessoa se comunicar genuinamente, e perceber-se como agente de resistência criativa ao encobrimento da sua condição de pessoa. Neste cenário, o medo de ser rejeitado pode ser superado, e a ansiedade deixar de ser sintoma, linguagem, estilo de viver e prisão para tornar-se autoconhecimento do sentido existencial da presença no mundo da vida. Compreender os significados da ansiedade coopera para que a pessoa unifique o arranjo pessoal da parte e do todo que abriga o (seu/nosso) viver, superando as dinâmicas defensivas de contato como guias crônicos. Conscientes que as emoções do medo, raiva, alegria, amor e o pensamento fazem parte da expressividade fecunda como qualidade da vida psíquica e social, a pessoa pode lidar com a ansiedade e ser inteira. 


\section{Referências}

Andreatini, R., Boerngen-Lacerda, R. \& Zorzetto Filho, D. (2001) Tratamento farmacológico do transtorno de ansiedade generalizada: perspectivas futuras. Brazilian Journal of Psychiatry. 23(4). 233-242. <https://doi.org/10.1590/S1516-44462001000400011>. https://doi.org/10.1590/S151644462001000400011

Andrade, J. V., et.al (2019). Ansiedade, um dos problemas do século XXI. Revista de Saúde da ReAGES, 2(4), 34-39.

Baiotto, C. S., Tremêa, G. T. F., \& Colet, C. de F. (2020), Propriedades farmacológicas atribuídas ao Rosmarinus Officinalis: uma revisão da literatura. Salão do conhecimento, UNIJUI.

Conceição, R. E. (2019). Potencial terapêutico da aromaterapia no manejo de transtornos de ansiedade. Monografia de Graduação em Farmácia, MG Universidade Federal de Ouro Preto.

Dalmarco, J. B. (2012). Estudo das propriedades químicas e biológicas de Rosmarinus officinalis L. Tese submetida ao Programa de Pós-graduação em Química da Universidade Federal de Santa Catarina para a obtenção do Grau de Doutor em Química na área de concentração Química Analítica. Florianópolis

Ehrenberg, A. (1991). Le culte de la performance. Philippe Fabry. Fiche de lecture du livre de Alain Ehrenberg:Le culte de la performance. de Alain Ehrenberg

Ehrenberg, A. (1995) L’individu incertain. Calmann-Lévy.

Ehrenberg, A. (1998). O cansaço de ser você mesmo - Depressão e sociedade, Odile Jacob, Paris 1998

Gouveia, G. D. A., \& Simionato, C. (2019). Memento fitoterápico para prática clínica na AB / Universidade Federal de Santa Catarina, Núcleo Telessaúde Santa Catarina. Florianópolis: CCS/UFSC.

Lima, Â. A., Heck, R. M., Barbieri, R. L., Portelinha, M. K., \& Lopes, A. C. P, (2016). Alternativas para a saúde: fitoterápicos populares produzidos pum grupo de agricultoras. Rev. enferm UFPE on line., 10(9):3320-8, set.

Lima, V. M. de. (2010). Avaliação da atividade antidepressiva e ansiolítica do óleo essencial de Rosmarinus officinalis L / Valéria Martins de Lima. Botucatu: [s.n.]

Macedo, B. K. (2011). O desamparo do indivíduo na modernidade. http://www.periodicoshumanas.uff.br/ecos

Melo, G. R. N. de, Nogueira, J. C. de C., Macêdo, A. E. G., Macêdo, C. G. (2020). Transtorno de ansiedade no interior da Amazônia: um estudo de base populacional Braz. J. of Develop., 6(1), 5301-5311.

Pimentel, A., Castro, E. H. B. de, \& Miranda, D (2019). Compreensão fenomenológica existencial da identidade de homens trans. ECOS | Estudos Contemporâneos da Subjetividade. 8(2). 229-239

Souza, J. A. de M. de \& Silva, C. de P. (2018). Chás e fitoterápicos indicados para distúrbios do sono, ansiedade e depressão, disponibilizados em estabelecimentos comerciais de São Caetano do Sul - SP. http://conic-semesp.org.br/anais/files/2018/trabalho-1000000560.pdf

Vanderlí, F. M. (2004). Monografia de Rosmarinus officinalis, - Fitomedicina Herbarium.

Pagani, C. A., \& Silva, B. F. da (2017). Uso popular de plantas medicinais no tratamento da ansiedade. http://www.uniedu.sed.sc.gov.br/wpcontent/uploads/2017/09/Claudia-Arruda-Pagani.pdf

Penteado, J. G., \& Cecy, A. T. (Alecrim Rosmarinus officinalis L. Labiatae (Lamiaceae): uma revisão bibliográfica http://www.unieuro.edu.br/sitenovo/ revistas/downloads/farmacia/cenarium_02_02.pdf

Zeni, F., Liz, M. P. de, Duarte, D., \& Zeni, A. L. B., (2021). Plantas medicinais e fitoterápicos na promoção à saúde no transtorno de ansiedade: uma revisão da literatura para apoio aos profissionais. Infarma, Ciências Farmacêuticas. 10.14450/2318-9312.v33.e1.a2021.pp6-17.

Pesquisa sobre ansiedade (2020). https://antigo.saude.gov.br/noticias/agencia-saude/47527-ministerio-da-saude-divulga-resultados-preliminares-depesquisa-sobre-saude-mental-na-pandemia 